\title{
\begin{tabular}{l|l} 
pcori $).$ & PATIENT-CENTERED OUTCOMES RESEARCH INSTITUTE \\
RESEARCH SUMMARY
\end{tabular}
}

\section{Improving Methods for Analyzing Data from Case-Only Studies}

Principal investigator

Murray A. Mittleman, MD, DPH
Organization

President and Fellows of Harvard College

\section{What was the project about?}

One way to see if a treatment works is to compare data from people who received the treatment with data from those who didn't or who received a different treatment. But sometimes the ways that people differ, such as their age or other health problems, can bias results. For example, if the people who didn't get the treatment are older or sicker than people who did get the treatment, results could suggest that the treatment works better than it really does.

One way to avoid this type of bias is to use case-only study designs. Case-only studies compare each patient's health before and after treatment. But caseonly studies often report the relative risk of a health event, such as stroke, among two groups of patients, instead of the absolute risk. For example, relative risk can show how the risk of stroke differs between patients who smoke and those who do not. Absolute risk would give the percentage of patients having a stroke among all patients. Absolute risk can help inform treatment decisions. But methods to measure absolute risk in case-only studies are limited. Also, clear guidance is lacking on how to best design and analyze a case-only study.

In this study, the research team created a guide and new methods for designing and analyzing case-only studies.

\section{What did the research team do?}

To create the guide for choosing the right study design, the research team compared six types of caseonly studies. First, the team used a computer program to create test data to look like real patient health data.
Using the test data, the team compared the accuracy of results from each of the six types of case-only studies to learn which study design works best for different types of data. The team also looked at the risk of bias under different scenarios. For example, in one scenario, patients' health habits, like smoking, changed before and after treatment.

The research team then created new methods to measure absolute risk in case-only studies. The methods show:

- How many people need to receive treatment for one of them to have a benefit or harm

- Whether treatments work better or worse for different groups of people

Doctors and patients provided input on the study design.

\section{What were the results?}

The research team created a guide to help researchers choose the right study design for case-only studies. The guide describes which case-only design to use based on the type of data and the purpose of the study. The new methods help measure absolute risk in case-only studies.

\section{What were the limits of the project?}

The new methods can only be used when patients' treatment status is a category, such as yes versus no or daily versus weekly. The methods don't work, for example, when a patient's treatment dose changes over time. 


\section{How can people use the results?}

The new methods can only be used when patients' treatment status is a category, such as yes versus no or daily versus weekly. The methods don't work, for example, when a patient's treatment dose changes over time.

To learn more about this project, visit www.pcori.org/Mittleman398. 